\title{
The Research of Long-Distance Data Transmission Based on Meteorological Sensor Network
}

\author{
Dehui Kong ${ }^{1,3}$, Tao Li ${ }^{1,3}$, Xingang You ${ }^{2,3}$, Xingming Sun ${ }^{1,3}$, \\ Baowei Wang ${ }^{1,3}$ and Qi Liu ${ }^{1,3}$ \\ ${ }^{1}$ Jiangsu Engineering Center of Network Monitoring, Nanjing University of \\ Information Science \& Technology, Nanjing, 210044, China \\ ${ }^{2}$ Beijing Institute of Electronic Technology Application, Beijing 100091, China \\ ${ }^{3}$ College of Computer \& Software, Nanjing University of Information Science \& \\ Technology, Nanjing, 210044, China \\ Correspondence should be addressed to Kong Dehui;bridgekdh@gmail.com
}

\begin{abstract}
Wireless sensor networks (WSNs) has gained more and more popularity in the field of intensive meteorological observation. Generally, each existing sensor node is a constrained device with restricted computation capability, and limited energy resources. The effective communication range of existing sensor nodes is unable to meet our requirements. In this paper, we present an improved system architecture of wireless sensor node that is capable of meeting the strict requirements of intensive meteorological observation systems. We add a solar power supply module to replenish node energy and adopt a network processor and a data acquisition processor collaborating with each other to improve the computation and computation capacity. Furthermore, we designed a dual-band data transmission mechanism so as to adapt to requirements of different transmission distances. Our tests have indicated that using solar power can fulfill a node's energy demand; whereas the design on computing and computing can dynamically meet the specific requirements of different applications. Moreover, our nodes is compatible with a variety of digital and analog sensor signal types, so it can be connected to various professional meteorological sensors directly. In addition, the communication distance of the node is significantly improved, which can reach more than 2000 meters in an open environment and 1000 meters with building obstruction or slight signal interference.
\end{abstract}

Keywords: Wireless sensor networks, meteorological observation, node, communication distance

\section{Introduction}

At present, the transmission medium of meteorological data is usually composed of wired and wireless. The wired transmission can realize the acquisition of meteorological data very well. But the deployment cost is relative high and cables are difficult to be laid in a complicated environment, e.g., mountains. Besides, it is very inconvenience to maintain and struggling to meet new application requirements [1]. Advances in hardware and wireless technologies have enabled the development of inexpensive low power communication devices deployed throughout a physical space, which can sense, process, communicate, and coordinate with each other. Compared with wired transmission manner, wireless sensor networks [2], which composed of a large number of these tiny devices, have many advantages in intensive meteorological observation. First, wireless communication makes the data no longer simply rely on cable transmission. Second, meteorological data collected by a large number of sensor nodes at the same time makes 
the collected data more concise. Last, it handles emergency events very well, e.g., earthquake or other disasters. Thus, WSNs are suitable for large-scale deployment. It can not only improve the precision and density in space and time of the meteorological observation but also can extend the existing intensive meteorological observation. Furthermore, it can be rapidly deployed when the infrastructure is lacked or destroyed and can provide emergency intensive meteorological services.

Currently available sensor nodes have some similarities in the structure, such as Berkeley Motes, Sensoria [3] WINS, MIT $\mu$ AMPs, InteliMote [4], Tmote [5], TinyNode [6], XYZ of the Yale University and smart-its BTNodes node [7] . Based on different application fields, these sensor nodes choose different core processor units and RF communication chips as described below. First is the selection of core processor units. MSP430 [8] microcontroller has the characteristics of ultra low power consumption, such as Tmote. But a node with a super processor is better at handling large amounts of data, suitable for high speed communication in a complex environment. And it has powerful data processing ability, such as Imote 2 and $\mathrm{XYZ}$ node [9]. It is a kind of preferred proposal that using ATMega128L chip processor with fast processing and low consumption. Second is the selection of RF communication chips. Nodes working at 2.4GHZ with 802.15.4 [10] communication protocols or Bluetooth communication protocols are widely used in wireless sensor networks. Its MAC communication protocol layer is solidified in the module, thus makes it simple to operate and does not need further development any more, such as Micaz [11], Tmote, Imote2 [12], XYZ node and BTNodes. But it has more advantages over communication distance when using other low frequency RF chips. In addition, a new MAC protocol can be developed to meet their requirements.

In order to realize the meteorological observation in WSNs, a series of challenges must be solved. First, due to the small size, sensor nodes usually carry limited energy, which is unrealistic to recharge or replace their batteries. Second, the computation and storage capacity of sensor nodes is also limited. A wireless sensor node is a tiny embedded device so that the node computation capability is weak and its storage capacity is relatively small. In order to accomplish various tasks, wireless sensor nodes need to monitor the data collection and data conversion, process the data and control the nodes to run. How to use the limited resources to complete the various tasks becomes a key concern in the design of wireless sensor nodes. In addition, wireless sensor nodes are faced with the interface mismatch problem when they are connected to professional meteorological sensors. Finally, the communication capability is limited. Many obstacles on the ground interfere with the node signal and interrupt the communication. Moreover, the existing wireless sensor nodes are unable to meet communication distance requirements of intensive meteorological observation.

In this paper, we combine the solar power with battery power to make up the limited energy resources. We designed the dual-processor to solve the limited storage capacity and computation speed. The core processor NANO130 with 32 bit ARM kernel is responsible for complex data collection and processing, and the processing speed can reach $100 \mathrm{MHz}$. The coprocessor ATMega128L with low power and 8 bit AVR kernel is responsible for data transmission. We provide the sampling function of RS485 signal type, SDI-12 signal type, differential analog signal type and digital signal type. Then wireless sensor node can be directly communicating with the meteorological sensors. In order to satisfy the requirements of meteorological data collection in transmit distance, we design a dual-band data transmission mechanism which combines $915 \mathrm{MHz}$ band with $2.4 \mathrm{GHz}$ band. The $915 \mathrm{MHz}$ module uses CC1101 as its RF chip and CC1190 as its RF front-end power amplifier. Its power is up to $25 \mathrm{dBm}$ and the highest sensitivity can be increased to $6 \mathrm{~dB}$. The effective communication distance can reach 3 kilometers. The 2.4GHz module adopts CC2520 chip as its RF chip and CC2591 as the front-end power amplifier. The output power is up to $22 \mathrm{dBm}$ and the effective communication distance is 
up to 1.5 kilometers. The wireless sensor node can switch the radio frequency band in time according to the surrounding environments to achieve different distance transmission.

In this paper we aim to design nodes that consume low power and have a lower overall cost when compared to commercially available ones. We have evaluated and selected components which fit our basic requirements of low cost, small size and high processing speed. The remainder of this paper is organized as follows: Section 2 explains the design of the hardware circuit. Section 3 deals with the software design of the nodes. Section 4 gives the performance test of this new WSN node. The paper is concluded in Section 5.

\section{The Design of Hardware Circuit}

\subsection{The Design of Meteorological Sensor Node}

1) The wireless sensor node consists of a sensor module, a processor module, a wireless communication module and a power supply module [13], as shown in Figure 1.

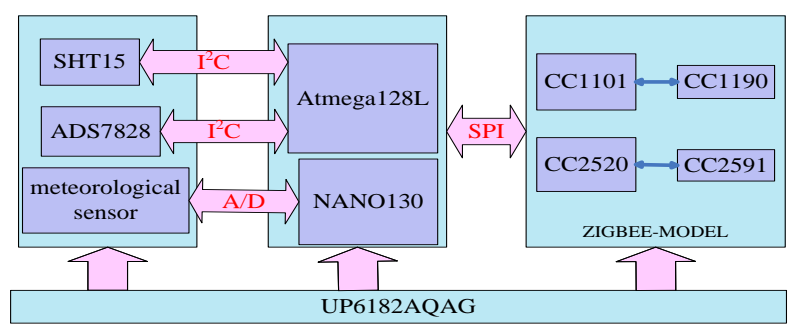

Figure 1. Internal Structure of the Node

The prototype of the new meteorological sensor node is shown in Figure 2.

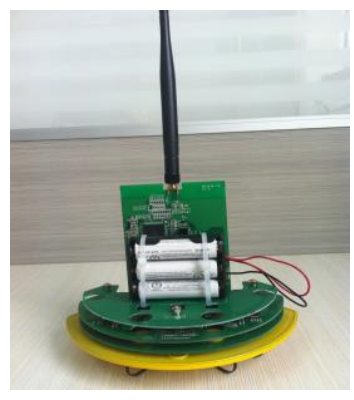

Figure 2. New Meteorological Sensor Node

2) The sensor module is composed of an internal temperature and humidity sensor SHT15 and professional meteorological sensors such as a temperature and humidity sensor CS215-L15, a Pyranometer CMP11, a wind direction sensor NRG \#200P and a wind speed sensor NRG \#40C. The sensor module collects the regional meteorological data and converts them in a suitable format.

3) The processor module is composed of a data acquisition chip NANO130 and a data transmission chip ATMega128L. They jointly control the node to store and process the transferred data and transfer them to the other nodes. NANO130 is connected with the professional meteorological sensors and collecting the sensor data. ATMega128L is connected with RF module and responsible for transmitting data.

4) The wireless communication module is composed of two parts. One part is $2.4 \mathrm{GHz}$ band communication equipped with $\mathrm{CC} 2520$ and CC2591. The other part is 
915MHz band communication equipped with CC1101 and CC1190. They can switch the radio frequency automatically based on the environment changes and the residual energy.

5) Energy supply module consists of two parts: One part is the three dry batteries. The other part is the solar energy power supply module. They provide the energy of the node together.

\subsection{ATmega128L connect with $2.4 \mathrm{GHz}$ [14]}

ATMega128L is connected with the Zigbee module, as shown in Figure 3.

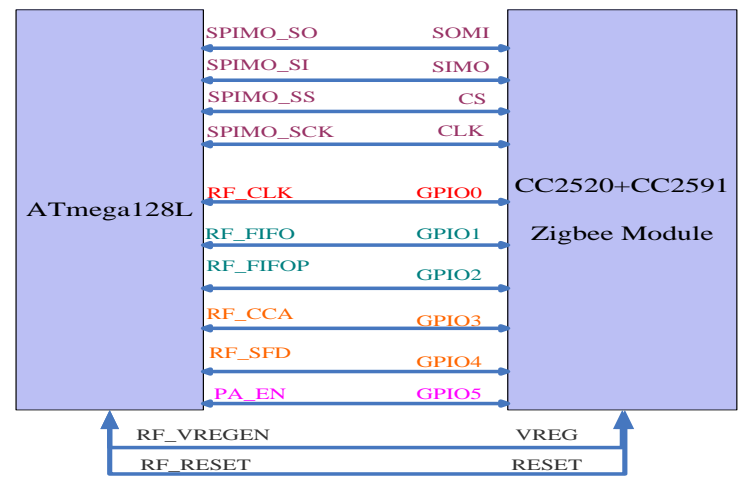

Figure 3. ATmega128 Connect with 2.4GHz Module

The connection of CC2520 [15] and ATMega128L [16] is very simple. CC2520 simulate four GPIO with six GPIO, including RF_FIFOP, RF_SFD, CCA and SFD. These pins indicate the sending or receiving data state. In the receiving mode, after receiving the separate delimiter of the start frame, the SFD pin is in high level until the frame is finish received. CCA is used to indicate if the current channel busy or not. CC2520 uses the RF_FIFO and RF_FIFOP pins to identify the RXFIFO state. If the RXFIFO received data, the RF_FIFO pin output high level. The FIFO pin output to low level if the RXFIFO underflow. The RF_FIFOP pin will output high level when the RXFIFO data exceeds a certain critical value or after CC2520 received a complete frame. It also output high level when RXFIFO underflows. ATMega128L exchanges data and control commands with CC2520 by SPI bus interface. ATMega128L access the CC2520's internal registers and memory through SPI bus and CC2520 act as the slave equipment, it receives the clock signal and the chip select signal from ATMega128L and executive input/output commands under control.

\subsection{ATmega128L Connect with $915 \mathrm{MHz}$}

ATMega128L is connected with $915 \mathrm{MHz}$, as shown in Figure 4.

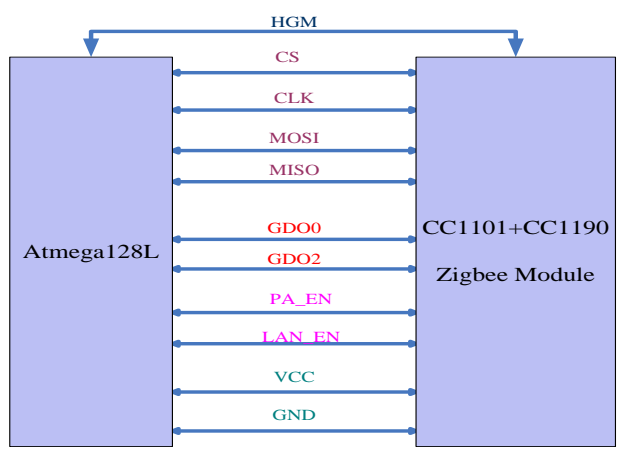

Figure 4. ATmega128 Connect with 915MHz Module 
Similar to ATmega128L and $2.4 \mathrm{GHz}$ communication module, ATmega128L access CC1101's internal registers and memory by SPI bus. Unlike the $2.4 \mathrm{GHz}$ band module, CC1101 [17] will generate an interrupt signal to MCU through GDO0 and GDO2. They can control software output useful internal state information.

\section{Software Design}

\subsection{The Data Collecting Design of NANO130}

The data collecting module is controlled by the NANO130 chip. The main function is to initialize the Watchdog, Power, SPI, $\mathrm{I}^{2} \mathrm{C}$, Led, Button, UART. Enable ADC interface, which will collect sensor data according to the data types and data length, store the collected data into the data buffer. As shown in Figure 5 is the working process of NANO130.

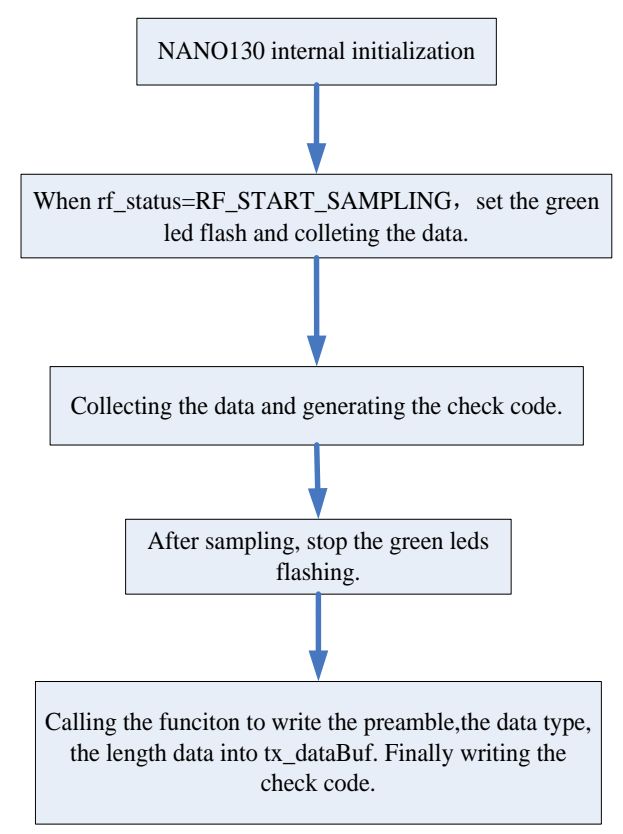

\section{Figure 5. Working Flow Chart of NANO130}

NANO130 collects sensor data through core_prepareSensorData() and generates check code through core_prepareTxChecksum(). After collected the data, calling the rf_sendSensorData() function to write the preamble, data types and data into tx_dataBuf through the UART port. Finally writes a check code and send out.

\subsection{The Design of a 915MHz ISM Band Transmission Module}

The $915 \mathrm{MHz}$ data transmission module is controlled by ATMega128L which was connected CC1101 and CC1190 through SPI bus. The sending and receiving data flow chart as shown below: 


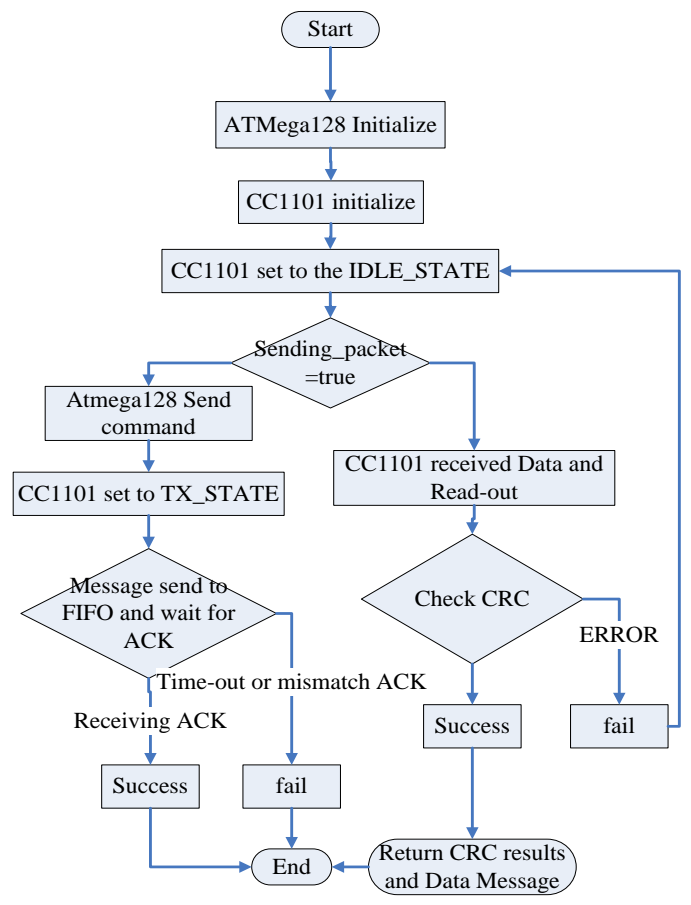

Figure 6. Data Transmission Flow Chart of $915 \mathrm{MHz}$

\subsection{The Design of a $2.4 \mathrm{GHz}$ ISM Band Transmission Module}

The $2.4 \mathrm{GHz}$ data transmission module is controlled by ATMega128L. It connects to CC2520 and CC2591 through SPI bus. The sending and receiving data flow chart as shown below:

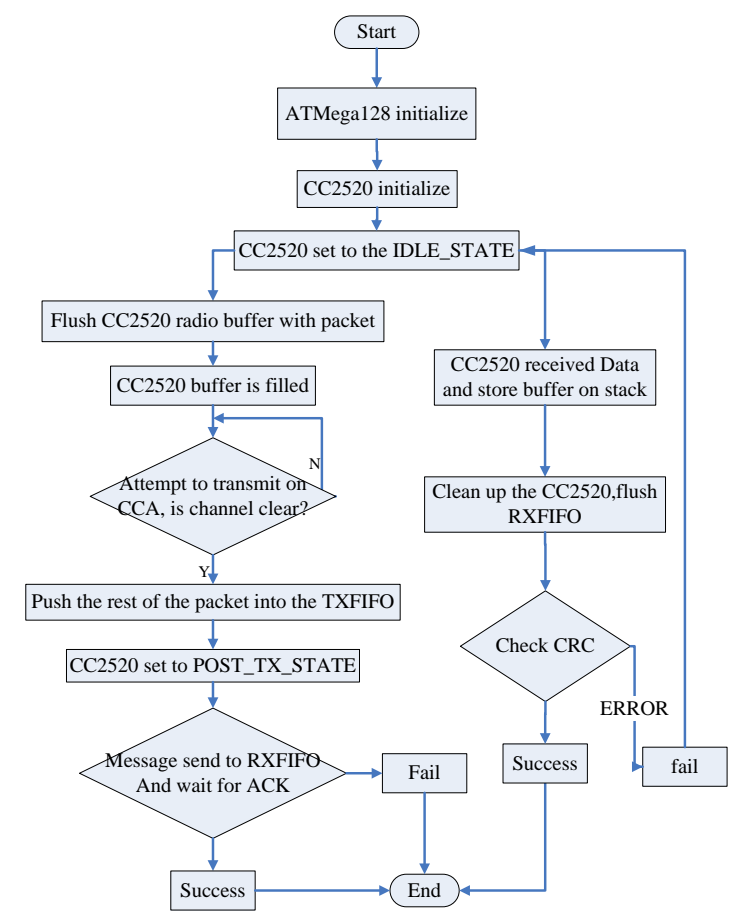

Figure 7. Data Transmission Flow Chart of $2.4 \mathrm{GHz}$ 


\section{Performance Test}

\subsection{The Solar Power Test}

The solar power module used a UP6182AQAG chip to charge to three AA batteries. We choose the Nanjing University of Information Science and Technology as the test place. The test time is $10 \mathrm{am}$ to $5 \mathrm{pm}$ of 2013-6-16. That day's minimum temperature is 26 degrees and the maximum temperature is 32 degrees.

The energy consumption diagram of data transmission in high power mode in the absence of solar powered module shows as below. X-axis represents the collected number of the voltage. Y-axis represents the voltage value of the node in real time.

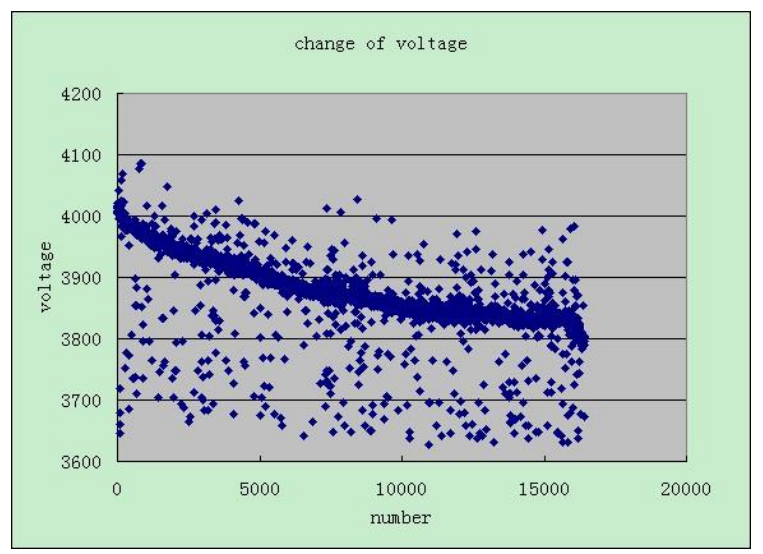

Figure 8. Energy Consumption in the Absence of Solar Powered Module

The energy consumption diagram of data transmission in high power mode in the charge of solar powered module shows as below:

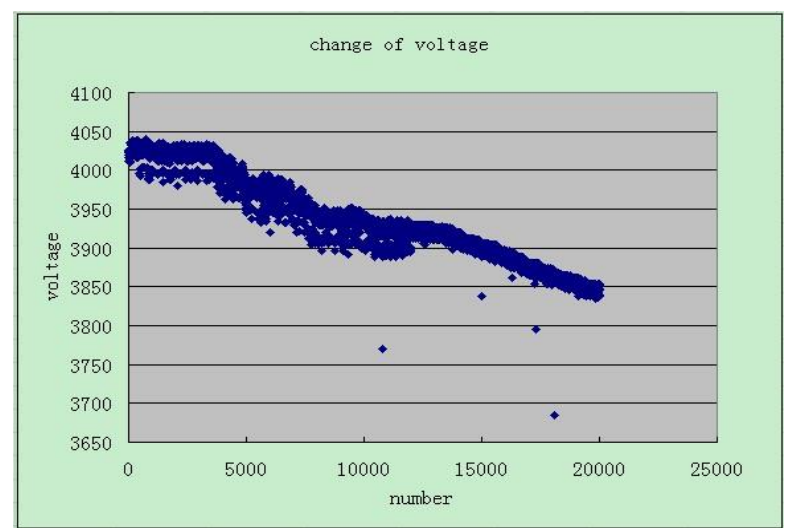

Figure 9. Energy Consumption in the Charge of Solar Powered Module

These two nodes located in the same place and set to launch in the same frequency. We can conclude two points according to the analysis above:

First, the power changed relatively stable in the charge of solar power.

Second, the voltage drop is relatively slower in the charge of solar power supplement.

\subsection{The Work Ability Test of the Node's Dual-processor}

According to the node in different working mode, we tested out the current consumption of the dual-processor in the following table: 
Table 1. Current Consumption under Different Working Mode

\begin{tabular}{|c|c|c|}
\hline Measurement scenario & $\begin{array}{c}\text { Measured current in } \\
\text { low power }\end{array}$ & $\begin{array}{c}\text { Measured current in } \\
\text { high power }\end{array}$ \\
\hline NANO130 in working mode & $10.6 \mathrm{~mA}$ & $20.5 \mathrm{~mA}$ \\
\hline NANO130 in idle mode & $4.6 \mathrm{~mA}$ & $14.5 \mathrm{~mA}$ \\
\hline NANO130 in power-down mode & $0.8 \mu \mathrm{A}$ & $1.2 \mu \mathrm{A}$ \\
\hline ATMega128L in working mode & $14.5 \mathrm{~mA}$ & $18 \mathrm{~mA}$ \\
\hline ATMega128L in idle mode & $6 \mathrm{~mA}$ & $8 \mathrm{~mA}$ \\
\hline ATMega128L in power-down mode & $8.2 \mathrm{uA}$ & $10.1 \mathrm{uA}$ \\
\hline
\end{tabular}

\subsection{The Test of Node's Signal Type}

The structure of the node is divided into two parts, including Header and Data. The length of the Header is 4 bytes, including board_id with one byte, packet_id with one byte and a parent_id with two bytes. The length of the Data is 38 bytes, including 16 bytes of ANALOG data type, 8 bytes of SDI-12 signal data type, the reserved 4 bytes of RS485 data type, 4 bytes of COUNTER data type, 2 bytes of TEMPERATURE data type, 2 bytes of HUMIDITY data [18] type and 2 bytes of BATTERY data type. The signal types include single-ended analog signal, differential analog signal, digital signal, the SDI-12 type signal and the RS485 type signal.

The colleted hexadecimal meteorological data and the converted data shown as figure 10.The wind value is named of "windir". The total value of radiation is named of "radiation". The wind speed is name of "winspd" and temperature and humidity represents the air temperature and humidity.

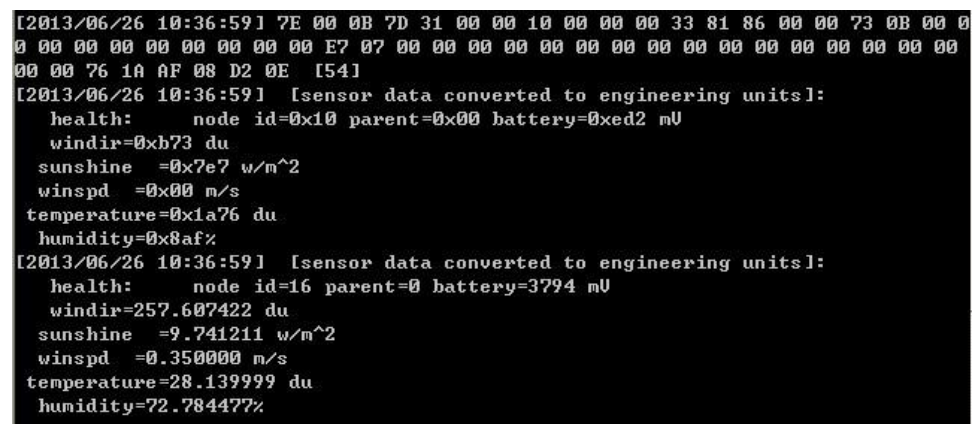

Figure 10. Raw Meteorological Data and the Converted Data

\subsection{The Communication Distance Test of $915 \mathrm{MHz}$ and $2.4 \mathrm{GHz}$ Dual-band Data Transmission}

Experiments were taken in the campus of Nanjing University of Information Science and Technology. The campus is full of French phoenix trees, as interference to the signal of nodes. The open environment is the straight distance between the building roofs. The occluded environment is created with the buildings and trees.

\section{1) Communication distance test of $2.4 \mathrm{GHz}$ module in opened environment [19]}

We located the sink nodes on a building roof. One observes the computer system and stand at the start point. The other one hold the node and move on towards an open field. Signals were emitted eight times per second. The test results are displayed in Table 2 
Table 2. Communication Distance of $2.4 \mathrm{GHz}$ Module in Opened Environment

\begin{tabular}{|l|c|c|c|c|c|}
\hline & 1 & 2 & 3 & 4 & 5 \\
\hline Distance $(\mathrm{m})$ & 842 & 840 & 835 & 829 & 820 \\
\hline
\end{tabular}

As shown in Table 2, stable data transmission at $2.4 \mathrm{GHz}$ in the open environment can be achieved within the distance of $820 \mathrm{~m}$. Repeated five times, an average distance of $833 \mathrm{~m}$ was obtained.

2) Communication distance test of $2.4 \mathrm{GHz}$ module in occluded environment

\section{Table 3. Communication Distance of 2.4GHz Module in Occluded} Environment

\begin{tabular}{|l|c|c|c|c|c|}
\hline & 1 & 2 & 3 & 4 & 5 \\
\hline Distance $(\mathrm{m})$ & 535 & 537 & 559 & 500 & 530 \\
\hline
\end{tabular}

As shown in Table 3 , data transmission at $2.4 \mathrm{GHz}$ in an occluded environment was stable in the distance of $500 \mathrm{~m}$. Repeated five times, an average distance $532 \mathrm{~m}$ can be obtained.

3) Communication distance test of $915 \mathrm{MHz}$ module in opened environment

\section{Table 4. Communication Distance of $915 \mathrm{MHz}$ Module in Opened} Environment

\begin{tabular}{|l|c|c|c|c|c|}
\hline & 1 & 2 & 3 & 4 & 5 \\
\hline Distance $(\mathrm{m})$ & 2020 & 2019 & 1989 & 2000 & 2010 \\
\hline
\end{tabular}

As shown in Table 4, data transmission at $915 \mathrm{MHz}$ in an opened environment was stable within the distance of 1989 meters. Repeated five times, an average distance of 2007 meters was obtained.

\section{4) Communication distance test of $915 \mathrm{MHz}$ module in occluded environment}

\section{Table 5. Communication Distance of $915 \mathrm{MHz}$ Module in Occluded Environment}

\begin{tabular}{|l|c|c|c|c|c|}
\hline & 1 & 2 & 3 & 4 & 5 \\
\hline Distance $(\mathrm{m})$ & 1010 & 1020 & 999 & 980 & 1022 \\
\hline
\end{tabular}

As shown in Table 5, data transmission at $915 \mathrm{MHz}$ in an occluded environment was stable in the distance of 980 meters. Repeated five times, an average distance 1006 meters was obtained.

\section{Conclusions and Future Work}

In this paper, we have presented the system architecture of a wireless sensor node that is capable of addressing the strict requirements of intensive meteorological observation. According to the testing results, the solar power supply meets the requirement of the nodes on energy consumption. The node's computation power can dynamically meet different application's needs. It can be connected to various professional meteorological sensors and can be used to collect the sensor data completely. In addition, the communication distance of the node is improved obviously. The data transmission distance can reach more than 2000 meters in an open environment and 1000 meters in an occluded environment. But the node still has some disadvantages. The energy is 
consumed more quickly than other nodes. The problem has been considered to solve in our next version of the design.

\section{Acknowledgements}

This work

is supported

by

the

NSFC(61232016,61173141,61173142,61173136,61103215,61373132,61373133),Nationa

1 Basic Research Program 973(2011CB311808),2011GK2009,GYHY201206033, 201301030,2013DFG12860,SBC201310569 and PAPD fund.

\section{References}

[1] I. F. Akyildiz, W. Su, Y. Sankarasubramaniam and E. Cayirci, "A Survey on Sensor Networks", Communications Magazine, IEEE, vol. 40, (2002).

[2] R. Min, M. Bhardwaj, S. Cho, E. Shih, A. Sinha, A. Wang and A. Chandrakasan, "Low-power wireless sensor networks", The 14th International Conference on VLSI Design, Bangalore, India, (2001)January.

[3] J. N. Al-Karaki and G. A. Al-Mashadbch, "SENSORIA: A New Simulatin Platform for Wireless Sensor Networks", International Conference on Sensor Technologies and Applications, Valencia, Spain, (2007) October14-20.

[4] L. Nachman, R. Kling, R. Adler, J. Huang and V. Hummel, "The Intel Mote Platform: A Bluetooth-Based Sensor Network for Industrial Monitiringm”, IPSN track on Sensor Platform Tools and Design Methods for Networked Embedded Systems, (2005).

[5] G. Werner-Allen, K. Lorincz, M. Ruiz, O. Marcillo, J. Johnson, J. Lees and M. Welsh, "Deploying a Wireless Sensor Network on an Active Volcano", Internet Computing, vol. 10, no. 2, (2006).

[6] H. Dubois-Ferriere, L. Fabre, R. Meier and P. Metrailler, "TinyNode: A Comprehensive Platform for Wireless Sensor Network Applications", Preceedings of the 5th international conference on Information processing in sensor networks, (2006) April19-21.

[7] J. Beutel, O. Kasten and M. Ringwald, "Poster abstract: BTodes-A Distributed Platform for Sensor Nodes", Proceedings of the1st international conference on Embedded networked sensor systems, New York, USA, (2003).

[8] MSP430Datasheet:http://sensorweb.cs.gsu.edu/sites/default/files/research/projects/TelosW/images/msp4 30F1611.pdf.

[9] D. Lymberopoulos and A. Savvides, "XYZ: A Motion-Enabled Power Aware Sensor Node Platform for Distributed Sensor Network Applications", Fourth International Symposium on Information Processing in Sensor Networks, (2005) April 15.

[10] IEEE 802.15.4 (2003):Wireless Medium Access Control (MAC) and Physical Layer (PHY) Specifications for Low-Rate Wireless Personal Area Networks (LR-WPANS).

[11] MICAZ Datasheet: http://www.openautomation.net/uploadsproductos/micaz_datasheet.pdf.

[12] L. Nachman, J. Huang, J. Shahabdeen, R. Adler and R. Kling, "IMOTE2: Serious Computation at the Edge”, Wireless Communications and Mobile Computing Conference, Crete Island, (2008) August 6-8.

[13] Y. Chengbo, C. Yanzhe, Z. Lian and Y. Shuqiang, "ZigBee Wireless Sensor Network in Environmental Monitoring Applications", WiCOM'09 Proceedings of the 5th International Conference on Wireless communications, networking and mobile computing, USA, (2009).

[14] N. S. Kulkarni, R. Rakesh, S. Bhargava, S. Singh Bundela and R. Hedge, "Zigbee Based Low Power Wireless Sensor Network Motes", 2010 International Conference on Next Generation Networks, Mumbai, (2010) September 24-25.

[15] CC2520 Datasheet: http://www.ti.com/lit/ds/symlink/cc2520.pdf.

[16] ATmega128L Datasheet: http://www.atmel.com/Images/doc2467.pdf.

[17] CC1101 Datasheet: http://www.ti.com/lit/ds/symlink/cc1101.pdf.

[18] Y. Zhou, Q. Zhou, Q. Kong and W. Cai, "Wireless Temperature \& Humidity Monitor and Control System”, 2012 2nd International Conference on Consumer Electronics, Communications and Networks (CECNet), Yichang, China, (2012) April21-23.

[19] C. Zhang, C. Li, S. Song and T. Hong, "Long-Distance Data Communication Based on Wireless Communication Technology", 2011 2nd International Conference on Artificial Intelligence, Management Science and Electronic Commerce (AIMSEC), Deng Leng, (2011) August 8-10. 


\section{Authors}

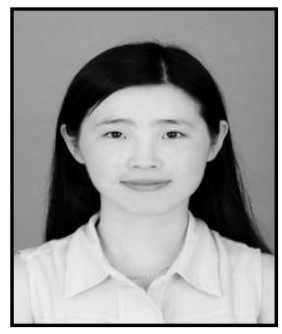

Dehui Kong, is a graduate student from Nanjing University of Information Science \& Technology currently. She received her bachelor degree in Computer Science \& Technology form Nanjing University of Information Science \& Technology in 2007. Her research interests mainly include wireless sensor networks.

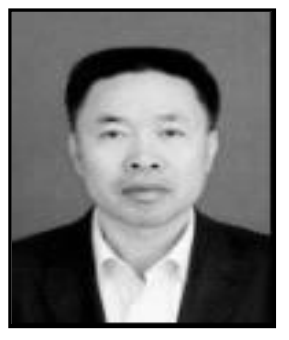

Xingming Sun, received his BS in mathematics from Hunan Normal University, China, in 1984, MS in computing science from Dalian University of Science and Technology, China, in 1988, and $\mathrm{Ph}$ in computing science from Fudan University, China, in 2001. He is currently a professor in School of Computer \& Software, Nanjing University of Information Science \& Technology, China. His research interests include network and information security, digital watermarking.

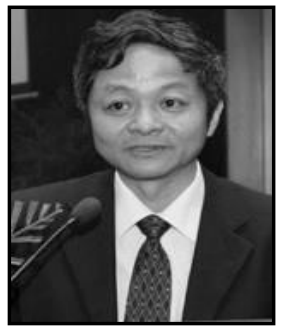

Xingang You, is a professor from Nanjing University of Information Science \& Technology, China. He received his Bachelor degree in Radio Engineering from Southeast University in 1984. Besides, he is also one of the directors of Chinese Computer Institute. His research area mainly covers steganography and Digital Watermarking technology signal processing and transmission technology and multimedia information security technology. He is now working as a doctoral supervisor in Beijing Institute of Electronic Technology. During his working time, he have undertaken many significant scientific researches, e.g. the national 863 project, and attained a lot of achievements. Plus, he also participated in the national 973 project and some natural science fund projects. In terms of honors, he attained multiple scientific researching achievements and was granted the national technical invention award and the scientific progress prize.

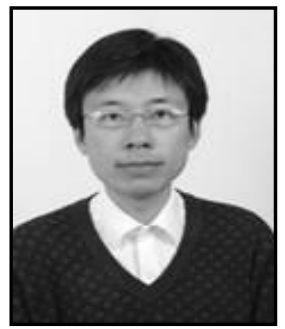

Tao Li, he received his MS degree in Computer Application from Nanjing University of Technology in 2004, and his PhD in Signal and Information Processing from Southeast University. He is currently working as a lecturer in School of Computer and Software, Nanjing University of Information Science and Technology. His research interests include wireless sensor network and embedded system. 


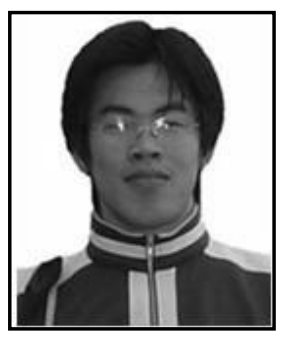

Baowei Wang, he received his B.S. and Ph.D. degrees in Computer Science from Hunan University in 2005 and 2011, respectively. He is currently working as a lecturer in School of Computer and Software, Nanjing University of Information Science and Technology. His research interests include steganography, wireless networks and securing adhoc networks.

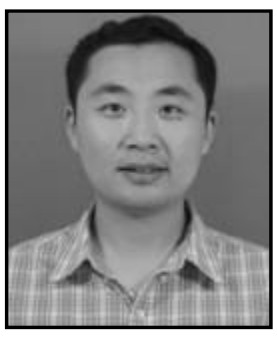

Qi Liu, he received his BS degree in computer Science and Technology from Zhuzhou Institute of Technology,China in2003, and his MS and $\mathrm{PhD}$ in Data Telecommunications and Networks from the University of Salford, UK in 2006 and 2010. His research interests include context awareness, data communication in MANET and WSN, and smart grid. His recent research work focuses on intelligent agriculture and meteorological observation systems based on WSN. 